\title{
No Ritmo de um Silêncio: a Música como Produtora de Processos Psicológicos
}

Ana Rita Monteiro Ribeiro*, Carlos Manuel Gonçalves**

\footnotetext{
* Mestre em Psicologia Clínica e da Saúde, Faculdade de Psicologia e Ciências da Educação na Universidade do Porto, Portugal. E-mail: ritamribeiro@live.com.pt

** Doutor em Psicologia, professor-auxiliar do departamento de Psicologia, Faculdade de Psicologia e Ciências da Educação, Universidade do Porto, Portugal. E-mail: carlosg@fpce.up.pt
}

Recibido: 29 de octubre del 2016 Aprobado: 7 de septiembre del 2017

Cómo citar este artículo: Ribeiro, A. R. M. e Gonçalves, C. M. (2017). No Ritmo de um Silêncio: a Música como Produtora de Processos Psicológicos. Pensando Psicología, 13(22), 61-75. doi: https://doi.org/10.16925/ pe.v13i22.1989

\section{Resumo}

Introdução: uma revisão teórica alargada em diversas áreas do saber fez antever a possibilidade de a música poder constituir um dispositivo privilegiado para a compreensão do desenvolvimento de estruturas psicológicas humanas e um motor propulsionador de mudanças. Objetivo: essa possibilidade permitiu configurar o seguinte objeto de estudo: compreender de que forma a música, enquanto arte, poderá possuir elementos potenciadores do desenvolvimento psicológico e emocional. Método: assim, desenhou-se um estudo que assumiu uma metodologia quantitativa a fim de avaliar o impacto da formação musical em três processos psicológicos: o processamento empático, a capacidade de diferenciação e a regulação emocional. Administraramse três instrumentos a uma amostra de 237 sujeitos: o Índice de Reatividade Interpessoal, a Escala de Avaliação do Repertório e da Capacidade de Diferenciação Emocional, e o Questionário de Regulação Emocional. Resultados: embora não se tenham verificado diferenças significativas entre ouvintes e performers, o presente estudo permitiu uma compreensão aprofundada sobre a forma como: o género pode determinar o processamento empático e o uso de estratégias adaptativas de regulação emocional; a progressão académica pode influenciar a forma de interpretar e agir sobre o meio envolvente. Conclusão: o envolvimento com a música, seja de que forma for, pode ser, por si só, suficiente para a promoção de determinados processos psicológicos.

Palavras-chave: diferenciação, empatia, ouvinte, performer, regulação emocional. 


\title{
To the Rhythm of Silence: Music as a Producer of Psychological Processes
}

\begin{abstract}
Introduction: An extended theoretical review of various fields of knowledge made us conceive the possibility that music could be a privileged device in understanding the development of human psychological structures and a driving force for change. Purpose: This possibility led us to outline the following objective: To understand how music, as an art, may possess elements that enhance psychological and emotional development. Method: Thus, a study was designed based on a quantitative methodology, in order to evaluate the impact of musical training on three psychological processes: empathic processing, differentiation ability, and emotion regulation. Three instruments were administered to a sample of 237 subjects: The Interpersonal Reactivity Index, the Emotion Differentiation Repertoire and Ability Assessment Scale, and the Emotion Regulation Questionnaire. Results: While there were no significant differences between listeners and performers, the study allowed an in-depth understanding of how gender can determine empathic processing and the use of adaptive emotion regulation strategies; academic progression can influence the way of interpreting and acting on the environment. Conclusion: Commitment to music, no matter how, can be sufficient to promote certain psychological processes.
\end{abstract}

Keywords: differentiation, empathy, listener, performer, emotion regulation.

\section{En el ritmo de un silencio: la música como productora de procesos psicológicos}

\section{Resumen}

Introducción: una revisión teórica ampliada en diversas áreas del saber hizo concebir la posibilidad de que la música pudiera constituir un dispositivo privilegiado en la comprensión del desarrollo de estructuras psicológicas humanas y un motor que promoviera cambios. Objetivo: esa posibilidad ha permitido configurar el siguiente objeto de estudio: comprender de qué forma la música, como arte, podrá poseer elementos potencializadores del desarrollo psicológico y emocional. Método: así, se diseñó un estudio basado en una metodología cuantitativa con el fin de evaluar el impacto de la formación musical en tres procesos psicológicos: el procesamiento empático, la capacidad de diferenciación y la regulación emocional. Se administraron tres instrumentos a una muestra de 237 sujetos: el Índice de Reactividad Interpersonal, la Escala de Evaluación del Repertorio y la Capacidad de Diferenciación Emocional, y la Encuesta de Regulación Emocional. Resultados: si bien no se han encontrado diferencias significativas entre oyentes y performers, el estudio ha permitido una comprensión profundizada acerca de la forma como el género puede determinar el procesamiento empático y el uso de estrategias adaptativas de regulación emocional; la progresión académica puede influir la forma de interpretar y actuar sobre el entorno. Conclusión: el compromiso con la música, no importa la forma, puede ser, de por sí, suficiente para la promoción de determinados procesos psicológicos.

Palabras clave: diferenciación, empatía, oyente, performer, regulación emocional. 


\section{Introdução}

Ouvir música, seja ela qual for e em que momento for, inevitavelmente desencadeia algum tipo de reação psicológica. A música que nos perpassa os ouvidos tem o condão de nos fazer arrepiar, rir ou chorar... Provoca a redução dos níveis de ansiedade, contribui para a diminuição da pressão arterial e da frequência cardíaca, modifica os nossos níveis de cortisol e de adrenalina no sangue, entre outras alterações fisiológicas.

$\mathrm{Na}$ literatura atual, são vários os estudos que sustentam os benefícios psicológicos que a música traz para os indivíduos (Sacks, 2007), uma vez que estimula a comunicação entre as pessoas e contribui de sobremaneira para o aumento da autoestima e da autoexpressão, podendo, aqui, ser mola de impulsão para a dança. A música é ainda capaz de promover momentos de reflexão ou de catarse, aumentando a capacidade de perceber e ouvir, de aprender, de ser social. É desta forma que a música e a psicologia avançam de mãos dadas. $\mathrm{Na}$ verdade, a psicologia tem dado as mãos a muitas e expressivas formas de arte porque a arte e a ciência são complementares e, desde os tempos mais idos, têm vindo a procurar formas de harmonização.

As artes são fontes de saber análogas às ciências e atuam de modos similares quanto às suas próprias construções. Goodman (2005) afina por esse diapasão garantindo que as artes não devem ser levadas menos a sério do que as ciências como modos de descoberta, criação e alargamento do conhecimento. É desta, cada vez mais, intrínseca relação entre a arte e a ciência que vão surgindo, ganhando forma e sendo cimentados os pilares em que a música se sustenta $\mathrm{e}$ de onde abraça, entre tantas outras, a possibilidade de atuar em conjunto com a psicologia, tocando emoções e trabalhando partituras que possam ser orquestradas em conjunto e em prol do bem comum.

A sinfonia que nasce deste dar de mãos entre a arte e a ciência, a música e a psicologia, ou entre a música e todas (ou quase todas) as áreas da saúde e do social chama-se musicologia. Esta é uma ciência que se dedica às questões teóricas e ao conhecimento alcançado nas várias áreas relacionadas à música. É, assim, uma disciplina vasta, que abriga diferentes ramos e perspetivas: a musicologia histórica explica o desenvolvimento da música ao longo dos séculos; a etnomusicologia estuda a música no contexto cultural em que se desenvolve; a teoria da música descreve os elementos da música, o desenvolvimento e o uso de métodos para compô-la e analisá-la.
Sacks (2007) adota o termo musicofilia para descrever que a propensão para a música que aparece na infância é visível e determinante em todas as culturas e, provavelmente, remonta aos começos da nossa espécie. Pode ser desenvolvida ou moldada pelas culturas em que vivemos, pelas circunstâncias da vida, ou pelos dons ou pelas fraquezas que cada um de nós tem, enquanto indivíduo - mas está tão fundamente enraizada em nós que somos tentados a encará-la como inata.

$\mathrm{Na}$ base desta dissertação, está latente a ideia de que a arte pode ser um meio eficaz de compreensão do funcionamento e das estruturas psicológicas humanas, bem como um meio terapêutico alternativo válido. A este respeito, Vygotsky (1971) afirma que a arte pode dar-nos informações sobre a forma como os indivíduos representam e compreendem a realidade, sendo um palco ideal para a construção de uma teoria do funcionamento psicológico.

São vastos e antigos os estudos que demonstram os efeitos da música em quase todos os aspetos das funções cerebrais - e na vida (Sacks, 2007). Este crescente campo de trabalho sobre os suportes neurais da perceção e da imagística musical são grandemente entusiasmantes e trazem cada vez mais credibilidade à aliança entre a música e a saúde mental. No entanto, não devemos esquecer que estudos sobre música e emoção correspondem apenas a uma das inúmeras subdimensões da musicologia, bem como a interface da música com outras áreas do saber.

Mais do que um mapa cerebral, a arte pode ser entendida como símbolos que refletem os propósitos cognitivos individuais; como aprendem, exploram e dão a conhecer o mundo (Goodman, 1978). Assim, a arte relaciona-se diretamente com a atividade humana de produção de significados: "Não só descobrimos o mundo através dos nossos símbolos como compreendemos e reavaliamos os nossos símbolos progressivamente à luz da nossa experiência precoce" (Goodman, 1978, p. 272). A produção de significados ocorre como resultado de interações com a arte, sobretudo na possibilidade de agir sobre ela ou participar dela. O indivíduo simultaneamente recebe a experiência e a constrói, sendo que a participação e a cocriação distanciam-se do deserto simbólico e da alienação.

Frisch, Franko e Herzog (2006) referem que o uso terapêutico de artes criativas promove o crescimento pessoal e a mudança positiva nos clientes. No entanto, já havia sido publicada uma teoria sobre temperamentos e sentimentos que defendia, sobretudo, que a personalidade do indivíduo estava relacionada 
com a resposta desse mesmo indivíduo aos estímulos musicais e que, por isso, a música se apresentava como excelente ferramenta quer para o tratamento de doenças (Carapetyan, 1948), quer para o desenvolvimento positivo de dimensões psicológicas.

Henderson e Gladding (1998) vêm mais uma vez reforçar o papel da arte ao afirmarem que esta pode: promover uma maior autoconsciência pela expressão simbólica; enfatizar e servir de suporte ao processo expressivo; permitir a partilha de experiências vivenciadas; promover sentimentos e afetos positivos, assim como lidar com os negativos; desenvolver novas ideias e formas mais criativas de relação com o mundo; promover a autoestima e desenvolver a capacidade de insight através da arte. A este propósito, Sacks (2007) afirma que a música parece de algum modo produzir um efeito nos humanos e ocupar um lugar central nas suas vidas. No entanto, não possui conceitos, não afirma nada, é desprovida de imagens ou símbolos, da substância da linguagem. Não se relaciona, necessariamente, com o mundo material e é evidente a emocionalidade que acarreta. A este respeito, Sprinthall (1991) preconiza que o envolvimento contínuo em experiências emocionalmente significativas favorece a transição para estádios de desenvolvimento mais complexos. Segundo Damásio (2003), a dimensão emocional é o meio natural de avaliar o ambiente que nos rodeia e de reagir de forma adaptativa. A criatividade assume aqui um importante papel de relevo (Goodman, 1978) e constitui-se como uma das funções basilares do funcionamento humano (Valquaresma e Coimbra, 2013).

A investigação por Ressonância Magnética funcional (fMRI) apresenta-se como uma ferramenta fundamental no estudo do cérebro humano. São vários os estudos que revelam que, quanto mais diversificada e emocionalmente carregada for a nossa ação/interação com o mundo, mais amplas e densas serão as sinapses das redes neuronais. Brembs, Ixirenzetti, Reys, Baxter e Byme (2002) sustentam a mesma ideia e acrescentam que estas redes neuronais traduzem-se no conhecimento e que as mudanças que aqui ocorrem representam a aprendizagem, possibilitando um maior desenvolvimento do sujeito (Zull, 2002).

De acordo com a investigação, as atividades artísticas comportam formas de produzir e integrar a experiência que estão mais próximas do modo como a investigação recente concebe o funcionamento psicológico, com vista ao desenvolvimento humano. Assim, se a arte promove novas formas de interpretar, compreender, conhecer e construir o mundo
(Goodman, 1978), o seu estudo e investigação poderão contribuir para o desenvolvimento de formas mais viáveis de pensar, sentir e agir (existir).

Ainda que esta complexidade de facetas e de estudos que a musicologia pressupõe possa, de algum modo, espaventar os estudiosos mais serenos, esta comporta vantagens significativas. A música apresenta-se como uma mais-valia e um motor propulsionador de mudanças pelo facto de envolver e afetar muitas facetas do ser humano e dada a diversidade das suas aplicações (Bruscia, 2000). Uma vez que este contributo de investigação se focaliza preferencialmente sobre três processos psicológicos, nomeadamente, a capacidade de diferenciação, a regulação emocional e a reatividade interpessoal (comummente denominada por empatia), é pertinente que se aprofunde a relação da música com estas dimensões psicológicas e emocionais.

Desde as civilizações mais antigas que os sons e a música têm a capacidade de comunicar emoções que podemos reconhecer, apreciar e sentir-nos movidos. Há assim evidências que permitem afirmar que ela assenta em alicerces biológicos que reforçam a sua validade enquanto objeto de estudo. As bases cognitivas e neuronais das emoções musicais é um tema que tem atraído as investigações mais recentes (Juslin, Liljeström, Västfjäll e Lundqvist, 2010; Juslin e Västfäll, 2008; Koelsch, 2010; Lima e Castro, 2011; Patel, 2008). As respostas de intenso prazer aos estímulos musicais envolvem sistemas neuronais de emoção e recompensa que respondem também a outros estímulos importantes para a sobrevivência biológica, tais como comida e sexo (Blood e Zatorre, 2001; Salimpoor, Benovoy, Larcher, Dagher e Zatorre, 2011). Estas evidências sugerem que o fenómeno essencialmente abstrato da música pode ter tido um papel neurobiológico durante a evolução humana, talvez por envolver a capacidade de resolução de problemas que facilita a descodificação de estados emocionais (Clark, Downey e Warren, 2014). Esta concepção sublinha ainda a função psicossocial da música, um tema-chave na neurociência contemporânea.

Esta forma de arte comunica emoções específicas (por exemplo, felicidade, tristeza, entre outras) de forma tão eficaz como outros estímulos socialmente proeminentes (como a prosódia da fala; Juslin e Laukka, 2003) e os mecanismos subjacentes ao reconhecimento emocional na música são robustos e de ação rápida: o reconhecimento ocorre com alta concordância dentro e entre os ouvintes (Bigand, Vieillard, Madurell, Marozeau e Dacquet, 2005). No que diz respeito ao processamento de emoções, a variabilidade é 
a regra, e não a exceção (Hamann, 2004). No entanto, os fatores que determinam a variabilidade no contexto de emoções musicais permanecem incompletamente compreendidos. Já tem sido várias vezes sustentado que a formação musical e o envelhecimento podem ter um amplo impacto nas funções neurocognitivas e socioemocionais (Dellacherie, Roy, Hugueville, Peretz e Samson, 2011; Hedden e Gabrieli, 2004; Samanez-Larkin e Carstensen, 2011).

Assim, no presente estudo, pretendemos examinar como e se a formação musical modula o processamento empático, a capacidade de diferenciação e a regulação emocional, sendo premente clarificar estes conceitos. As emoções não se resumem à reação fisiológica relativamente a um estímulo, mas são constituídas por uma multiplicidade de processos que as caracterizam. Como resposta a um determinado estímulo (interno ou externo), o ser humano experiencia uma determinada reação fisiológica (ativação emocional) que, posteriormente, através da atribuição de um significado (diferenciação emocional), se diferencia e ganha coerência. Desta forma, as emoções surgem quando um estímulo é avaliado como significativo. No contacto com a música, a emoção é, na maior parte das vezes, ativada automaticamente, e são vários os autores que defendem esta premissa, ainda que atribuam a ativação emocional a diferentes componentes musicais (ritmo, harmonia, melodia, entre outros).

Além de desencadear emoções, a música é também um veículo usado para expressar sentimentos, sobretudo, através da empatia musical. Se, por um lado, as emoções nos permitem organizar e interpretar o meio que nos envolve e agir sobre ele, a empatia é o que nos permite sentir o que outra pessoa está a sentir, saber o que outra pessoa está a sentir e responder à experiência de outrem (Decety, 2004). Assim, o processo empático acontece quando a observação de alguém desencadeia mecanismos que produzem respostas no observador (Limpo, Alves e Castro, 2013). Segundo os estudos de Zahn-Waxler, Radke-Yarrow e Brady-Smith (1977), a empatia envolve não só a capacidade cognitiva, mas sobretudo emocional, sendo por isso um processo indissociável das emoções.

Estudos científicos comprovam que o cérebro não dispõe de um "centro musical", mas ativa uma ampla gama de áreas para interpretar os diferentes timbres, dinâmicas e ritmos, e realizar a descodificação métrica e melódica, bem como a modulação do sistema de prazer e recompensa envolvido na experiência musical. O processamento da música começa com a penetração das vibrações sonoras no ouvido interno, provocando movimentos nas células ciliadas que variam de acordo com a frequência das ondas. Os estímulos sonoros seguem pelo nervo auditivo até ao lobo temporal, onde se dá a perceção sensorial da musical: é nesta fase que são descodificados o timbre, a dinâmica, a melodia e o ritmo do som. O lobo temporal interliga-se em circuitos de ida e volta com o hipocampo, uma das áreas ligadas à memória, o cerebelo e a amígdala, áreas que integram o chamado cérebro primitivo e são responsáveis pela regulação motora e emocional, e ainda um pequeno núcleo de massa cinzenta, relacionado à sensação de bem-estar gerada pela música. Assim, esta ativação cerebral concebe a comunicação musical como uma forma de empatia (Forgeard, Winner, Norton e Schlaug, 2008). É também importante referir que a estimulação externa a que estamos expostos modela a nossa atividade cerebral uma vez que o cérebro tem necessidade de se adaptar ao meio ambiente, aumentando a plasticidade cerebral, assim como a capacidade adaptativa (Pascual-Leone, Amedi, Fregni e Merabet, 2005). Esta sensibilidade cerebral aumenta com a experiência musical que cada indivíduo tem.

Evidências crescentes sugerem que o treino musical beneficia a codificação neuronal. Neste sentido, Patel (2011) desenvolveu a Opera Hypothesis que propõe que tais benefícios sejam movidos pela plasticidade adaptativa nas redes de processamento da fala, e que essa plasticidade ocorre quando são cumpridas determinadas condições, das quais se destacam a emoção, a atenção e a repetição. De acordo com esta hipótese, quando estas condições forem satisfeitas, as redes de plasticidade neuronal funcionam com maior precisão do que o necessário para a comunicação comum. Assim, os músicos apresentam maiores volumes em áreas específicas do cérebro, uma vez que o treino musical exige uma maior adaptação cerebral à exigência que lhe é colocado.

Por fim, chegamos ao cerne desta investigação: se o processamento musical pressupõe empatia e se a empatia envolve a capacidade emocional, então, indivíduos com formação musical e/ou performers/compositores terão índices mais elevados em ambos os processos quando comparados a ouvintes.

É neste contexto exploratório que surge esta investigação que visa compreender como o estímulo de arte musical desencadeia processos psicológicos emocionais de empatia, de diferenciação e regulação emocional. Desta forma, ensaia-se dar resposta à questão central de investigação: os sujeitos com formação musical e/ou performers/compositores terão 
níveis mais elevados de empatia e de diferenciação e regulação emocional quando comparados com sujeitos meramente ouvintes musicais?

Assim, os objetivos da presente investigação sumarizam-se nos seguintes tópicos:

- compreender de que forma a música pode influenciar direta ou indiretamente o processamento empático, a diferenciação e a regulação emocional;

- verificar se o processamento empático, a diferenciação e a regulação emocional variam em função da forma como os indivíduos se relacionam com a música, ou seja, ser mero ouvinte musical ou performer/compositor;

- verificar se existirão diferenças de género e nível de escolaridade relativas às dimensões psicológicas da empatia da diferenciação e da regulação emocional, quer em ouvintes, quer em performers/ compositores.

\section{Método}

\section{Participantes}

Neste estudo, participaram 273 sujeitos com idades compreendidas entre 16 e 29 anos. Para a seleção da amostra, foram tidos como critérios de inclusão: ser apenas ouvinte ou performer/compositor. Conforme apresentado na Tabela 1, a amostra total é constituída por 102 elementos do género masculino (37.4\%) e 171 do género feminino (62.6\%). Os participantes do género masculino apresentam a idade mínima de 18 e máxima de $28(M=23.66$; $D P=2.52)$ e o género feminino a idade mínima de 16 e a máxima de $29(M=22.37 ; D P=2.60)$. Da amostra total, 93 sujeitos têm habilitações literárias até ao ensino secundário (34.1\%) e 180 até ao ensino superior (65.9\%). Relativamente à música, 146 sujeitos consideram-se apenas ouvintes (53.5\%) e 127 consideram-se perfomers/compositores (46.5\%), sendo que 81 sujeitos têm formação musical (29.7 \%). Por fim, 122 sujeitos são instrumentistas (44.7\%), sendo que a guitarra clássica é o instrumento musical predominante.

\section{Materiais}

Os dados tratados neste estudo foram recolhidos através de um questionário composto por questões sociodemográficas (idade, género, habilitações literárias e atividade profissional) e questões que pretendiam
Tabela 1

Caracterização da amostra

\begin{tabular}{lccc}
\hline & $n(\%)$ & $\begin{array}{c}\text { Média } \\
(D P)\end{array}$ & $\begin{array}{c}\text { Min.- } \\
\text { Máx. }\end{array}$ \\
\hline Idade & \multirow{2}{*}{271} & $\begin{array}{c}22.85 \\
(2.64)\end{array}$ & $16-29$ \\
\hline
\end{tabular}

\section{Género}

$\begin{array}{lll}\text { Feminino } & 171(62.6 \%) & \begin{array}{l}22.37 \\ (2.60)\end{array} \\ & & 23.66 \\ \text { Masculino } & 102(37.4 \%) & (2.52)\end{array}$

\section{Habilitações literárias}

$\begin{array}{lc}\text { Ensino secundário } & 93(34.1 \%) \\ \text { Ensino superior } & 180(65.9 \%)\end{array}$

Ensino superior $\quad 180(65.9 \%)$

\begin{tabular}{lccc}
\hline Relativamente à música & & & \\
Apenas ouvinte & $146(53.5 \%)$ & & \\
Performer/compositor & $127(46.5 \%)$ & & \\
\hline Formação musical & & & \\
Sim & $81(29.7 \%)$ & & \\
Não & $192(70.3 \%)$ & & \\
Duração & 73 & 9.26 & $1-20$ \\
Instrumento musical & & & \\
Sim & $122(44.7 \%)$ & & \\
Não & $151(55.3 \%)$ & & \\
Duração & & 8.42 & $0-23$ \\
\hline
\end{tabular}

Nota. Elaboração própria

recolher algumas informações referenciadas com o estudo a desenvolver, como se são meros ouvintes ou performers/compositores, se têm formação musical e qual o instrumento musical que tocam.

O protocolo de avaliação global incluiu os instrumentos a seguir.

- O Î́ndice de Reatividade Interpessoal (IRI) (Davis, 1980; 1983, versão portuguesa de Limpo, Alves e Castro, 2013) foi utilizado com o objetivo de avaliar a empatia. O IRI é constituído por 24 itens, organizados em quatro subescalas: Tomada de perspetiva $(\alpha=.73)$, Preocupação empática ( $\alpha=.76)$, Desconforto pessoal $(\alpha=.80)$ e Fantasia $(\alpha=.84)$. A dimensão cognitiva é apreciada através da Tomada de perspetiva, e a dimensão afetiva, pelas restantes subescalas. 
- A Escala de Avaliação do Repertório e da Capacidade de Diferenciação Emocional (EARCDE) (Kang e Shaver, 2004, versão portuguesa de Vaz e Martins, 2009) avalia o repertório e a capacidade do indivíduo para experienciar e diferenciar emoções. Na sua forma original, a EARCDE é uma medida de autorrelato que foi desenvolvida com o objetivo de avaliar a natureza e as características dos indivíduos no âmbito da complexidade emocional. A escala é constituída por 14 itens, organizados em duas subescalas: Reportório emocional $(\alpha=.63)$ e Diferenciação emocional $(\alpha=.82)$.

- O Questionário de Regulação Emocional (QRE) (Gross e John, 2003, versão portuguesa de Vaz e Martins, 2009) é um método de avaliação de estratégias de regulação emocional e de compreensão das diferenças individuais na utilização destas estratégias em situações específicas. O QRE é constituído por 10 itens, organizados em duas subescalas: Reavaliação cognitiva $(\alpha=.76)$ e Supressão emocional $(\alpha=.65)$.

As respostas obtidas em cada instrumento foram cotadas numa escala tipo Likert entre 1 e 6, em que 1 significa "Discordo totalmente", e 6, "Concordo totalmente".

\section{Procedimentos}

O procedimento de recolha de dados decorreu ao longo de dois meses, com os respetivos consentimentos dos autores de cada instrumento utilizado. Os questionários foram entregues e preenchidos presencialmente com duração de cerca de 12 minutos.

Para satisfazer alguns requisitos, no que respeita à distribuição da amostra, foram contactados alguns grupos e instituições que pudessem fornecer dados de sujeitos com e/ou sem formação musical. O caráter voluntário da participação foi assegurado com todos os participantes, assim como a confidencialidade dos dados. Foi sempre dada a oportunidade de colocar dúvidas relacionadas ao estudo ou ao preenchimento do questionário.

\section{Análise de dados}

Após a recolha, obteve-se a análise dos dados por estatística descritiva e inferencial através do Statistical Package for Social Sciences (sPss), versão 22.0. A normalidade encontra-se assegurada uma vez que, em grandes amostras ( $\mathrm{n}>30)$, as análises estatísticas são suficientemente robustas para suportar a violação do pressuposto da normalidade (Pallant, 2011). Desta forma, foram utilizados testes paramétricos, nomeadamente, a análise da variância multivariada (manova) e o Coeficiente de Correlação de Pearson.

\section{Resultados}

O objetivo deste estudo foi investigar a relação entre a formação musical e as dimensões psicológicas: reatividade interpessoal, diferenciação e regulação emocional.

Não foram encontradas diferenças estatisticamente significativas entre ouvintes e performers/ compositores relacionadas às escalas IRI $(U=8760$; $p=.560)$, EARCDE $(U=8839 ; p=.645)$ e QRE $(U=8445$; $p=.334$ ). No que diz respeito às subescalas, foram apenas encontradas diferenças estatisticamente significativas entre ouvintes e performers/compositores quanto ao Reportório emocional $(U=7889 ; p=.43)$.

Na MANova, podemos verificar diferenças estatisticamente significativas associadas ao género $F$ $(8,257)=5.885, p<.001$; Wilk's $\Lambda=.845$, partial $\eta 2=.155$. Podemos verificar nos dados apresentados na Tabela 2 o efeito estatisticamente significativo do género no IRI $(F(8,257)=29.855, p<.001$; partial $\eta 2=.102)$ e nas subescalas da Preocupação empática $(F(8,257)=24.444, p<.001$; partial $\eta 2=.085)$, do Desconforto pessoal $(F(8,257)=12.777, p<.001$; partial $\eta 2=.046)$, da Fantasia $(F(8,257)=17.597$, $p<.001$; partial $\eta 2=.062)$ e da Supressão emocional $(F(8,257)=5.809, p=.017$; partial $\eta 2=.022)$, cujos valores médios se apresentam superiores no género feminino, exceto na subescala da Supressão emocional, cuja média é superior no género masculino.

De acordo com os dados apresentados na Tabela 3 , podemos verificar diferenças estatisticamente significativas no IRI $(F(8,257)=4.659, p=.032$; partial $\eta 2=.017)$, na subescala Tomada de perspetiva $(F(8,257)=9.751, p=.002$; partial $\eta 2=.036)$, Fantasia $(F(8,257)=5.419, p=.021$; partial $\eta 2=.020)$, na EAR$\operatorname{CDE}(F(8,257)=5.917, p=.016$; partial $\eta 2=.022)$ e na subescala Repertório emocional $(F(8,257)=4.769$, $p=.030$; partial $\eta 2=.018$ ), cujos valores médios se apresentam superiores nos sujeitos com o ensino superior quando comparados com os do ensino secundário.

Na Tabela 4, estão apresentadas as associações do Coeficiente de Correlação de Pearson entre as subescalas de cada instrumento em que se verificou uma correlação significativa positiva forte entre a Fantasia e a Preocupação empática $(r=.556 ; p<.01)$, e entre 
Tabela 2

Média, desvio-padrão e nível de significância da MANOva dos instrumentos e das subescalas por género

\begin{tabular}{|c|c|c|c|c|c|}
\hline & $\begin{array}{c}\text { Feminino } \\
(n=169)\end{array}$ & $\begin{array}{l}\text { Masculino } \\
\quad(n=97)\end{array}$ & & & \\
\hline & Média (DP) & Média (DP) & $F$ & $p$ & $\eta 2$ \\
\hline IRI & $3.98(.47)$ & $3.65(.50)$ & $29.855^{\star * \star}$ & .000 & .102 \\
\hline Tomada de perspetiva & $4.28(.65)$ & $4.25(.74)$ & .166 & .684 & .001 \\
\hline Preocupação empática & $4.44(.74)$ & $3.97(.80)$ & $24.444^{* * *}$ & .000 & .085 \\
\hline Desconforto pessoal & $3.26(.77)$ & $2.89(.75)$ & $12.777^{\star \star *}$ & .000 & .046 \\
\hline Fantasia & $3.94(.86)$ & $3.48(.96)$ & $17.597^{\star * *}$ & .000 & .062 \\
\hline EARCDE & $4.15(.75)$ & $4.06(.68)$ & 1.290 & .257 & .005 \\
\hline Repertório emocional & $4.03(1.05)$ & $3.84(.92)$ & 2.413 & .122 & .009 \\
\hline Diferenciação emocional & $4.28(.78)$ & $4.28(.75)$ & .014 & .905 & .000 \\
\hline QRE & $3.58(.74)$ & $3.61(.78)$ & .134 & .715 & .001 \\
\hline Reavaliação cognitiva & $4.00(1.08)$ & $3.80(1.03)$ & 1.878 & .172 & .007 \\
\hline Supressão emocional & $3.16(.76)$ & $3.41(.94)$ & $5.809^{* * *}$ & .017 & .022 \\
\hline
\end{tabular}

Nota. ${ }^{* * *} \mathrm{p}<.001$. IRI (Índice de Reatividade Interpessoal); EARCDE (Escala de Avaliação do Repertório e da Capacidade de Diferenciação Emocional); QRE (Questionário de Regulação Emocional); DP (Desvio-padrão). Elaboração própria

Tabela 3

Média, desvio-padrão e nível de significância da MANOVA dos instrumentos e das subescalas por habilitações literárias

\begin{tabular}{|c|c|c|c|c|c|}
\hline & $\begin{array}{l}\text { Ensino secundário } \\
\qquad(n=93)\end{array}$ & $\begin{array}{c}\text { Ensino superior } \\
\quad(n=180)\end{array}$ & & & \\
\hline & Média (DP) & Média (DP) & $\mathrm{F}$ & $p$ & $\eta 2$ \\
\hline IRI & $3.77(.54)$ & $3.90(.48)$ & $4.659^{* * *}$ & .032 & .017 \\
\hline Tomada de perspetiva & $4.11(.77)$ & $4.35(.62)$ & $9.751^{\star * *}$ & .002 & .036 \\
\hline Preocupação empática & $4.19(.81)$ & $4.31(.79)$ & 2.315 & .129 & .009 \\
\hline Desconforto pessoal & $3.20(.82)$ & $3.08(.77)$ & 1.913 & .168 & .007 \\
\hline Fantasia & $3.62(.89)$ & $3.85(.93)$ & 5.419 & .021 & .020 \\
\hline EARCDE & $3.99(.68)$ & $4.18(.74)$ & $5.917^{\star * *}$ & .016 & .022 \\
\hline Repertório emocional & $3.82(.93)$ & $4.03(1.04)$ & 4.769 & .030 & .018 \\
\hline Diferenciação emocional & $4.17(.76)$ & $4.33(.77)$ & 2.965 & .086 & .011 \\
\hline QRE & $3.60(.81)$ & $3.58(.73)$ & .019 & .891 & .000 \\
\hline Reavaliação cognitiva & $3.89(1.14)$ & $3.95(1.03)$ & .299 & .585 & .001 \\
\hline Supressão emocional & $3.32(.82)$ & $3.22(.85)$ & .882 & .349 & .003 \\
\hline
\end{tabular}

Nota. ${ }^{* * *} \mathrm{p}<.001$. IRI (Índice de Reatividade Interpessoal); EARCDE (Escala de Avaliação do Repertório e da Capacidade de Diferenciação Emocional); QRE (Questionário de Regulação Emocional); DP (Desvio-padrão). Elaboração própria.

o Repertório emocional e a Preocupação empática $(r=.547 ; p<.01)$. Encontraram-se correlações significativas positivas moderadas entre a Preocupação empática e a Tomada de perspetiva $(r=.303 ; p<.01)$, entre o Repertório emocional e a Fantasia $(r=.443$; $p<.01)$, entre a Diferenciação emocional e a Tomada de perspetiva $(r=.362 ; p<.01)$, entre a Diferenciação emocional e o Repertório emocional $(r=.307 ; p<.01)$ e entre a Reavaliação cognitiva e a Tomada de perspetiva $(r=.316 ; p<.01)$. 
Tabela 4

Coeficiente de Correlação de Pearson entre as subescalas de cada instrumento

\begin{tabular}{|c|c|c|c|c|c|c|c|c|}
\hline & \multicolumn{3}{|c|}{ IRI } & \multicolumn{2}{|c|}{ EARCDE } & \multicolumn{3}{|c|}{ QRE } \\
\hline & TP & PE & DP & $\mathbf{F}$ & $\mathbf{R E}$ & DE & $\mathbf{R C}$ & SE \\
\hline \multicolumn{9}{|l|}{ IRI } \\
\hline \multicolumn{9}{|l|}{$\mathrm{TP}$} \\
\hline $\mathrm{PE}$ & $.303^{* *}$ & & & & & & & \\
\hline $\mathrm{DP}$ & $-.191^{* *}$ & .080 & & & & & & \\
\hline $\mathrm{F}$ & $.193^{* *}$ & $.556^{* *}$ & $.184^{* *}$ & & & & & \\
\hline \multicolumn{9}{|c|}{ EARCDE } \\
\hline $\mathrm{RE}$ & $.260^{* *}$ & $.547^{* *}$ & $.153^{*}$ & $.443^{* *}$ & & & & \\
\hline $\mathrm{DE}$ & $.362^{* *}$ & $.236^{* *}$ & -.033 & $.251^{* *}$ & $.307^{* *}$ & & & \\
\hline \multicolumn{9}{|c|}{ QRE } \\
\hline $\mathrm{RC}$ & $.316^{* *}$ & $.140^{*}$ & -.073 & .086 & .087 & $.215^{* *}$ & & \\
\hline $\mathrm{SE}$ & $.151^{*}$ & -.056 & -.063 & $-.134^{*}$ & -.086 & .099 & $.244^{* *}$ & \\
\hline
\end{tabular}

Nota. ${ }^{\star} \mathrm{p}<.05 ;{ }^{* *} \mathrm{p}<.01$. IRI (Índice de Reatividade Interpessoal); TP (Tomada de Perspetiva); PE (Preocupação Empática); DP (Desconforto Pessoal); F (Fantasia); earcde (Escala de Avaliação do Repertório e da Capacidade de Diferenciação Emocional); RE (Repertório Emocional); DE (Diferenciação Emocional); QRE (Questionário de Regulação Emocional); RC (Reavaliação Cognitiva); se (Supressão Emocional). Elaboração própria

$\mathrm{Na}$ Tabela 5, estão apresentadas as associações do Coeficiente de Correlação de Spearman entre as escalas e as subescalas de cada instrumento e a idade, sendo que apenas se encontraram correlações significativas fracas.

\section{Discussão}

Antes de fazer qualquer interpretação dos resultados, é importante relevar que, trabalhando com uma ferramenta de autorrelato, ou seja, uma ferramenta que, no fundo, avalia as percepções de cada indivíduo acerca das suas capacidades, exige-se um certo distanciamento porque, na realidade, existe uma certa margem de erro em relação aos resultados reais. Como não se está a realizar uma investigação laboratorial objetivante ou a aceder aos significativos e às narrativas estruturantes dos indivíduos, é importante ter em mente que os testes podem conter enviesamentos marcados pela desejabilidade social subjacente a esta tipologia de instrumentos utilizada nas metodologias de investigação quantitativa, como é o caso (Howard e Dailey, 1979).

Sumarizando, os principais resultados deste estudo sugerem que:

1) relativamente ao género, as mulheres têm maior tendência para adotar o ponto de vista do outro, maior
Tabela 5

Coeficiente de Correlação de Spearman entre as escalas $e$ as subescalas de cada instrumento e a idade

\begin{tabular}{lcc}
\hline & \multicolumn{2}{c}{ Idade } \\
\hline & $\mathbf{R}_{\mathrm{s}}$ & $\mathbf{P}$ \\
\cline { 2 - 3 } IRI & -.019 & .762 \\
$\mathrm{TP}$ &. $\mathbf{1 8 2 ^ { * * }}$ & $\mathbf{. 0 0 3}$ \\
$\mathrm{PE}$ & .041 & .499 \\
$\mathrm{DP}$ & $-.19^{* *}$ & $\mathbf{. 0 0 2}$ \\
\hline $\mathrm{F}$ & -.075 & .220 \\
EARCDE &. $\mathbf{1 7 6 ^ { * * }}$ & $\mathbf{. 0 0 4}$ \\
$\mathrm{RE}$ & $\mathbf{. 1 2 3 ^ { * }}$ & $\mathbf{. 0 4 3}$ \\
$\mathrm{DE}$ &. $\mathbf{1 5 6 ^ { * }}$ & $\mathbf{. 0 1 0}$ \\
$\mathbf{Q R E}$ & .025 & .687 \\
$\mathrm{RC}$ & .020 & .739 \\
$\mathrm{SE}$ & -.001 & .982 \\
\hline
\end{tabular}

Nota. ${ }^{*} \mathrm{p}<.05 ;{ }^{*} \mathrm{p}<.01$. IRI (Índice de Reatividade Interpessoal); тP (Tomada de Perspetiva); PE (Preocupação Empática); DP (Desconforto Pessoal); F (Fantasia); EARCDE (Escala de Avaliação do Repertório e da Capacidade de Diferenciação Emocional); RE (Repertório Emocional); DE (Diferenciação Emocional); QRE (Questionário de Regulação Emocional); RC (Reavaliação Cognitiva); se (Supressão Emocional). Elaboração própria 
capacidade para experienciar sentimentos de compaixão e preocupação pelo outro e maior capacidade de avaliar sentimentos de ansiedade, apreensão e desconforto em contextos interpessoais tensos;

2) por sua vez, os homens têm maior tendência para suprimir a expressão emocional;

3) em relação à formação académica, os sujeitos de formação superior têm maior capacidade empática, repertório e capacidade de experienciar e diferenciar emoções quando comparados com os estudantes do secundário;

4) os ouvintes têm maior capacidade de avaliar diferenças subtis entre emoções similares e maior variedade de emoções experienciadas em relação aos performers/compositores;

5) a capacidade empática assim como as estratégias de diferenciação e regulação emocional aumentam com a idade;

6) no entanto, os sentimentos de ansiedade, apreensão e desconforto em contextos interpessoais tensos diminuem com a idade.

Os resultados encontrados, quanto às diferenças de género, no processamento empático, são consistentes com os de estudos anteriores, nomeadamente com os resultados obtidos na adaptação do IRI à população portuguesa, que apontam para a superioridade no grupo feminino nas várias subescalas (Davis, 1980, 1983; Limpo, Alves e Castro, 2013). Quanto à EARCDE, o género não teve qualquer impacto em nenhuma das variáveis estando também de acordo com os resultados da adaptação da escala à população portuguesa. No entanto, no QRE, verificou-se que os homens utilizam mais a supressão emocional que as mulheres (que utilizam mais a reavaliação cognitiva, embora sem resultados significativamente estatísticos).

A este propósito, Gross e John (2003) defendem que indivíduos que utilizam a reavaliação cognitiva como estratégia de regulação emocional experienciam e expressam mais emoções positivas e menos emoções negativas. De acordo com a literatura, no âmbito afetivo, a supressão emocional e a reavaliação cognitiva têm consequências afetivas diferentes. A supressão diminui a expressão de emoções desadaptativas e cria um sentimento de discrepância entre o que foi experienciado e o que o indivíduo mostra aos outros, conduzindo a emoções negativas acerca de si próprio. Em situações extremas, este processo pode aumentar a alienação do indivíduo perante os outros, impedindo o desenvolvimento de relações emocionalmente próximas (John e Gross, 2004 citado em
John e Gross, 2007). Assim, indivíduos que frequentemente utilizam a supressão como estratégia de regulação emocional experienciam menos emoções positivas. Estes resultados poderão ser interpretados pelo facto de as mulheres serem, por processos educativos e culturais, mais sensíveis às dimensões emocionais nas relações interpessoais do que os homens, que privilegiam as dimensões marcadas pela instrumentalidade e pela racionalidade.

No que diz respeito às habilitações literárias, os resultados obtidos demonstraram diferenças entre os grupos em comparação. Este impacto verificouse nos resultados obtidos no IRI, nomeadamente na subescala da tomada de perspetiva e na EARCDE. Desta forma, sujeitos com o ensino superior parecem ter melhor processamento empático, nomeadamente, uma maior tendência para adotar o ponto de vista do outro, assim como maior repertório e capacidade de experienciar e diferenciar emoções. Uma vez mais, estes resultados vão ao encontro dos resultados obtidos na adaptação da EARCDE à população portuguesa, que concluíram que a progressão académica aumenta o repertório emocional e cognitivo dos indivíduos (Vaz e Martins, 2009).

Também o padrão de correlações encontrado no IRI foi, de forma geral, semelhante ao encontrado na literatura, definindo a empatia como um conjunto de fenómenos diferenciáveis entre si, mas associados por serem aspetos da reação do próprio enquanto observador de outrem (Limpo, Alves e Castro, 2013).

Os resultados de comparação do grupo de ouvintes com o grupo de performers/compositores vão de encontro aos observados na comparação do grupo de instrumentistas e não instrumentistas. A comparação entre ouvintes e performers/compositores não foi, até à data, estudada aprofundada e/ou diretamente e, portanto, não é possível realizar uma interpretação sustentada de confrontação dos resultados deste estudo devido à escassez de literatura no que respeita às dimensões analisadas.

Não obstante, nos últimos anos, têm surgido novas evidências de que tocar um instrumento está relacionado com melhores índices de função executiva (Bialystok e DePape, 2009; Bugos, Perlstein, McCrae, Brophy e Bedenbaugh, 2007; Collins, 2013). Uma vez que a função executiva é responsável por processos relativos a tarefas de planeamento e capacidade de resolução de problemas (Collins, 2013), seria de esperar que indivíduos que tocam um instrumento musical, quando comparados com não instrumentistas, demonstrassem diferenças substanciais no 
que diz respeito à diferenciação e à regulação emocional. No entanto, tal não se verificou, uma vez que não se atingiram resultados de significância satisfatórios em nenhum dos instrumentos. Analisando mais de perto estes resultados, talvez seja possível identificar alguns aspetos acerca do porquê de não terem surgido diferenças significativas entre estes grupos.

No que concerne à reatividade interpessoal e assumindo que o IRI avalia a empatia através das dimensões cognitiva e afetiva, não foram encontrados estudos que fossem ao encontro da relação destas capacidades com a música. Não obstante, considerou-se que a capacidade de tentar compreender sentimentos e emoções era uma condição necessária na presença de resultados significativos de outras medidas que já foram abordadas na literatura.

A correlação existente entre os instrumentos utilizados permitiu concluir que, para um indivíduo saber diferenciar o que está a sentir, é necessário estar atento não só às suas emoções, mas também às emoções dos outros. Especificamente no domínio interpessoal, a capacidade de diferenciação emocional potencia a compreensão empática dos outros (Kang e Shaver, 2004), na medida em que se constitui como um meio de informação acerca do que os outros poderão experienciar durante uma relação interpessoal. Consequentemente, esse processo fortalece o estabelecimento de relações mais positivas. Resultados de investigações recentes demonstraram ainda que indivíduos com uma maior capacidade de diferenciação emocional apresentam maior diversidade e adequabilidade de estratégias de regulação emocional (Barrett, Gross, Christensen e Benvenuto, 2001). Indivíduos que se descrevem a si próprios com elevada diferenciação emocional (definida como a capacidade de ter grande diversidade de experiências emocionais bem diferenciadas) são mais atentos aos seus sentimentos, mais abertos à experiência e mais empáticos em relação aos outros, mostrando, por isso, maior adaptabilidade interpessoal (Kang e Shaver, 2004).

Van Goethem e Sloboda (2011) concluíram que o ato de ouvir música oferece maior leque de estratégias de regulação emocional eficazes. Os resultados deste estudo refletem que, o facto de os sujeitos se envolverem mais ou menos com a música (ser ouvinte ou performer/compositor e instrumentista ou não instrumentista) pode não ter influência, interessando apenas que haja contacto com a música. Assim, e considerando a literatura em geral, é importante esclarecer o que contribui na música para esse desenvolvimento, uma vez que diferentes autores colocam focos diferentes nos processos ou nas tarefas musicais em estudo. Isto é, os benefícios da música para o desenvolvimento de determinados processos psicológicos podem dever-se ao envolvimento ativo com ela (Hallam, 2010b), apenas ao ato de ouvir música (Shifriss, Bodner e Palgi, 2014), ao benefício de tocar um instrumento (Patel, 2011), à educação musical (Collins, 2013) ou até mesmo ao treino musical (Corrigall, Schellenberg e Misura, 2013). Ao serem abordados de forma diferente, a concordância dos seus resultados pode gerar confusão, uma vez que não se compreende o que beneficia o quê $\mathrm{e}$ a preponderância das dimensões ativadas no desenvolvimento psicológico. Estas conclusões tratam-se apenas de conjeturas, sendo que o problema da escassez de investigação acerca das dimensões em estudo continua a ser uma realidade. Devemos ter presente, uma vez mais, que os resultados refletem a autoperceção dos indivíduos sobre as suas capacidades empáticas e emocionais, e não a capacidade real, podendo enviesar, por isso, os resultados. A ausência de diferenças pôde ainda resultar da desejabilidade social e fazer com que os indivíduos respondessem intencionalmente de forma não genuína.

Os resultados relativos à idade revelaram-se significativos, embora o intervalo da amostra total fosse curto (treze anos), já de forma a evitar enviesamentos que pudessem advir do aumento da idade. De modo geral, todas as dimensões psicológicas avaliadas parecem obter melhores resultados de acordo com o aumento da idade, sobretudo no IRI e na EARCDE. Assim, a idade correlaciona-se positivamente com a tomada de perspetiva e negativamente com o desconforto pessoal, o que, de acordo com Piaget (2008), acontece devido ao processo de desenvolvimento das estruturas cognitivas e emocionais. O ser humano está sempre em transformação passando por várias experiências, assimilando-as e acomodando-as de acordo com suas necessidades e as oportunidades desafiantes proporcionadas ou não pelos seus contextos de vida. A maturação é, assim, um processo essencial no desenvolvimento individual e garante o amadurecimento psicológico. Desta forma, é natural que as capacidades intelectuais dos indivíduos melhorem com o decorrer do tempo, com o investimento na formação e com novas experiências acumuladas de exploração do mundo. Carstensen et al. (2011) demonstraram também que o domínio emocional se torna mais adaptativo com a idade e que o controlo emocional evolui.

Em face aos resultados apresentados e discutidos, sublinhe-se, mais uma vez, que o presente estudo 
assume um carácter eminentemente exploratório e visa ser um modesto contributo que coloca prioritariamente desafios à investigação futura, mais do que o propósito de apresentar grandes conclusões.

\section{Conclusão}

Nos últimos anos, tem-se verificado um crescimento significativo de estudos no domínio dos diversos processos emocionais, sobretudo, no âmbito internacional. Em Portugal, são ainda escassos, particularmente sobre instrumentos de avaliação validados para a população portuguesa, dificultando, por isso, a realização de investigações neste domínio do saber. Apesar de se levantarem diversas questões sobre a validade do constructo quando estas dimensões são avaliadas através de medidas de autorrelato, os resultados da investigação prévia demonstram que, quando os indivíduos completam medidas de autocaracterização da capacidade emocional, têm que recordar, sumariar e integrar a sua experiência passada num conjunto consistente de respostas. Desta forma, as medidas de autorrelato são consideradas válidas como qualquer outro instrumento de avaliação, desde que sustentadas teoricamente e com caraterísticas psicométricas consistentes, podendo ser retirada e utilizada como válida a informação psicológica que fornecem.

Os instrumentos utilizados, embora recentemente adaptados à população portuguesa, permitiram uma compreensão mais profunda sobre a forma como o género pode determinar o processamento empático assim como o uso de estratégias adaptativas ou desadaptativas de regulação emocional; o modo como a progressão académica pode influenciar a forma de organizar e interpretar o meio que nos envolve e agir sobre ele, e, sobretudo, compreender a relação ativa ou o envolvimento com a experiência musical na promoção de determinados processos psicológicos.

Não obstante, o presente estudo apresenta várias limitações:

- quanto à metodologia adotada: ao optar-se por uma metodologia de cariz quantitativa focalizada em representações, pelo recurso a instrumentos de autorrelatos, corre-se o risco de as representações serem contaminadas pela desejabilidade social;

- a opção pelas variáveis estudadas de cariz eminentemente emocional como o processamento empático, a capacidade de diferenciação e a regulação emocional, omitindo variáveis de cariz mais cognitivo, como o desenvolvimento de estruturas cognitivas de ação, tomada da perspetiva social, relacionamento interpessoal, entre outras, limita a compreensão do impacto da música no desenvolvimento global do sujeito;

- a ausência de algumas variáveis no questionário sociodemográfico, nomeadamente as áreas de estudo dos participantes com formação superior, não permitiu a análise de possíveis relações ou comparações com os processos psicológicos em estudo; - a dificuldade de encontrar instrumentos, adaptados à população portuguesa, para avaliar o impacto da música no desenvolvimento de processos psicológicos condicionou a escolha das variáveis a estudar. E, ainda, os instrumentos utilizados não estão suficientemente desenvolvidos, em virtude de terem sido recentemente adaptados à população portuguesa e não terem sido testados em várias investigações;

- uma outra limitação prende-se com a constituição da amostra de pura conveniência. A opção por uma amostra homogeneizada relativamente à faixa etária mais jovem, não só ao nível dos ouvintes e performers/compositores ou instrumentistas e não instrumentistas, bem relativamente ao género e à área geográfica onde foram recolhidos os dados, circunscrevendo-se à Região do Grande Porto;

- por fim, salienta-se a escassez de investigação no âmbito internacional e, sobretudo, nacional sobre o objeto de estudo abordado, dificultando a interpretação dos resultados encontrados.

Em face às limitações sinalizadas, propõe-se para futuras investigações, que se intensifiquem estudos nesta área do saber alargando as variáveis psicológicas em análise, pelo recurso a metodologias mais complexas, de caráter misto (qualitativas e quantitativas), em que as representações sejam replicadas pelo confronto com os significados através da análise dos discursos e das narrativas dos intervenientes.

Dada a recente adaptação dos instrumentos utilizados nesta investigação, sugere-se, em futuros estudos, que se realize uma nova análise fatorial exploratória dos três instrumentos em ordem a afinar e desenvolver o rigor das várias subescalas, para aumentar a qualidade psicométrica deles, como os valores de Alfa de Cronbach de consistência interna de cada subescala e a percentagem da variância explicada. Após este trabalho parcimonioso, num segundo momento, propõe-se que se realizem as respetivas análises fatoriais confirmatórias para validar os modelos teóricos subjacentes aos instrumentos.

Por fim, mais do que os resultados conseguidos, esta investigação permitiu explorar o impacto da música 
no desenvolvimento humano e o encanto que esta tem para todo aquele que se envolve com ela. Tendo em conta os benefícios da música para o desenvolvimento pessoal e emocional, a introdução precoce desta forma de arte no sistema educativo parece ser uma boa forma de combater todas as vulnerabilidades com que os indivíduos se deparam nos dias de hoje e, sobretudo, uma forma de promover o desenvolvimento humano global. A este propósito, são vários os países que têm adotado a música como parte integrante dos planos curriculares, sendo os seus resultados positivos e irrefutáveis (Hallam, 2010a). Assim, estas propostas não se traduzem numa solução, mas, como o presente estudo sugere, um meio de promover processos psicológicos de forma saudável e criativa.

\section{Referências}

Barrett, L., Gross, J., Christensen, T. e Benvenuto, M. (2001). Knowing what you're feeling and knowing what to do about it: Mapping the relation between emotion differentiation and emotion regulation. Cognition and Emotion, 15(6), 713-724. doi: 10.1080/02699930143000239.

Bialystok, E. e DePape, A. (2009). Musical expertise, bilingualism, and executive functioning. Journal of Experimental Psychology: Human Perception and Performance, 35(2), 565-574. doi: 10.1037/a0012735.

Bigand, E., Vieillard, S., Madurell, F., Marozeau, J. e Dacquet, A. (2005). Multidimensional scaling of emotional responses to music: The effect of musical expertise and of the duration of the excerpts. Cognition and Emotion, 19(8), 1113-1139. doi: 10.1080/02699930500204250.

Blood, A. e Zatorre, R. (2001). Intensely pleasurable responses to music correlate with activity in brain regions implicated in reward and emotion. Proceedings of the National Academy of Sciences, 98(20), 11818-11823. http://dx.doi.org/ 10.1073/pnas.191355898.

Brembs, B., Ixirenzetti, F. D., Reys, F. D., Baxter, D. A. e Byme, J. H. (2002). Operant reward learning in aplysia: Neuronal correlates and mechanisms. Science, 296(5573), 1706-1709. doi: 10.1126/science.1069434.

Bruscia, K. E. (2000). Definindo a musicoterapia. Rio de Janeiro: Ene livros.

Bugos, J., Perlstein, W., McCrae, C., Brophy, T. e Bedenbaugh, P. (2007). Individualized piano instruction enhances executive functioning and working memory in older adults. Aging \& Mental Health, 11(4), 464-471. doi: 10.1080/13607860601086504.

Carapetyan, A. (1948). Music and Medicine in the Renaissance and in the 17th and 18th Centuries. Em D. M.
Schullian e M. Schoen (Eds.), Music and Medicine (pp. 117-157). Nova York: H. Wolff.

Carstensen, L., Turan, B., Scheibe, S., Ram, N., ErsnerHershfield, H., Samanez-Larkin, G., Brooks, K. e Nesselroade, J. (2011). Emotional experience improves with age: Evidence based on over 10 years of experience sampling. Psychology and Aging, 26(1), 21-33.

Clark, C., Downey, L. e Warren, J. (2014). Brain disorders and the biological role of music. Social Cognitive and Affective Neuroscience, 10(3), 3-17. doi: 10.1093/scan/nsu079.

Collins, A. (2013). Neuroscience meets music education: Exploring theimplications ofneural processingmodelsonmusic education practice. International Journal of Music Education, 31(2), 217-231. doi: 10.1177/0255761413483081.

Corrigall, K., Schellenberg, E. e Misura, N. (2013). Music training, cognition, and personality. Frontiers in psychology, 4, 1-9. doi: 10.3389/fpsyg.2013.00222.

Damásio, A. (2003). Ao encontro de Espinosa. Lisboa: Publicações Europa-América.

Davis, M. H. (1980). A multidimensional approach to individual differences in empathy. JSAS Catalog of Selected Documents in Psychology, 10, 85-103. Recuperado de https://goo.gl/d3yV3g.

Davis, M. H. (1983). Measuring individual differences in empathy: Evidence for a multidimensional approach. Journal of Personality and Social Psychology, 44(1), 113-126.

Decety, J. (2004). The functional architecture of human empathy. Behavioral and Cognitive Neuroscience Reviews, 3(2), 71-100. doi: 10.1177/1534582304267187.

Dellacherie, D., Roy, M., Hugueville, L., Peretz, I. e Samson, S. (2011). The effect of musical experience on emotional self-reports and psychophysiological responses to dissonance. Psychophysiology, 48(3), 337-349. doi: 10.1111/j.1469-8986.2010.01075.x.

Forgeard, M., Winner, E., Norton, A. e Schlaug, G. (2008). Practicing a Musical Instrument in Childhood is Associated with Enhanced Verbal Ability and Nonverbal Reasoning. PLOS ONE, 3(10), e3566.

Frisch, M., Franko, D. e Herzog, D. (2006). Arts-based therapies in the treatment of eating disorders. Eating Disorders, 14(2), 131-142. doi: 10.1080/10640260500403857.

Goodman, N. (1978). Ways of worldmaking. Indianápolis: Hackett Pub. Co.

Gross, J. e John, O. (2003). Individual differences in two emotion regulation processes: Implications for affect, relationships, and well-being. Journal of Personality and Social Psychology, 85(2), 348-362. doi: 10.1037/0022-3514.85.2.348. 
Hallam, S. (2010a). Music education: the role of affect. Em P. N. Juslin e J. A. Sloboda (eds.), Handbook of music and emotion (pp. 791-818). Oxford: Oxford University Press.

Hallam, S. (2010b). The power of music: Its impact on the intellectual, social and personal development of children and young people. International Journal of Music Education, 28(3), 269-289. doi: 10.1177/0255761410370658.

Hamann, S. (2004). Individual differences in emotion processing. Current Opinion in Neurobiology, 14(2), 233 238. doi:10.1016/j.conb.2004.03.010.

Hedden, T. e Gabrieli, J. (2004). Insights into the ageing mind: a view from cognitive neuroscience. Nature Reviews Neuroscience, 5(2), 87-96. doi: 10.1038/nrn1323.

Henderson, D. e Gladding, S. (1998). The creative arts in counseling: a multicultural perspective. The Arts in Psychotherapy, 25(3), 183-187. doi: 10.1016/s01974556(98)00011-2.

Howard, G. e Dailey, P. (1979). Response-shift bias: A source of contamination of self-report measures. Journal of Applied Psychology, 64(2), 144-150. doi: 10.1037//00219010.64.2.144.

John, O. P. e Gross, J. J. (2007). Individual differences in emotion regulation. Em J. Gross (ed.), Handbook of emotion regulation (pp. 351-372). Nova York: Guilford Press.

Juslin, P. e Laukka, P. (2003). Communication of emotions in vocal expression and music performance: Different channels, same code? Psychological Bulletin, 129(5), 770-814. doi: 10.1037/0033-2909.129.5.770.

Juslin, P. e Västfäll, D. (2008). Emotional responses to music: The need to consider underlying mechanisms. Behavioral and Brain Sciences, 31(05). doi: 10.1017/s0140525x08005293.

Juslin, P., Liljeström, S., Västfäll, D. e Lundqvist, L. (2010). How does music evoke emotions? Exploring the underlying mechanisms. Em P. N. Juslin e J. A. Sloboda (eds.), Handbook of music and emotion (pp. 605-642). Oxford: Oxford University Press.

Kang, S. e Shaver, P. (2004). Individual differences in emotional complexity: Their psychological implications. Journal of Personality, 72(4), 687-726. doi: 10.1111/j.0022-3506.2004.00277.x.

Koelsch, S. (2010). Towards a neural basis of music-evoked emotions. Trends in Cognitive Sciences, 14(3), 131-137. doi: 10.1016/j.tics.2010.01.002.

Lima, C. e Castro, S. (2011). Speaking to the trained ear: Musical expertise enhances the recognition of emotions in speech prosody. Emotion, 11(5), 1021-1031. doi: $10.1037 / \mathrm{a} 0024521$.
Limpo, T., Alves, R. e Castro, S. (2013). Medir a empatia: Adaptação portuguesa do Índice de Reactividade Interpessoal. Laboratório de Psicologia, 8(2). doi: 10.14417/lp.640.

Pallant, J. (2011). spss Survival Manual: A step by step guide to data analysis using spss. $4^{\text {a }}$ ed. Crows Nest Nsw: Allen \& Unwin.

Pascual-Leone, A., Amedi, A., Fregni, F. e Merabet, L. (2005). The Plastic Human Brain Cortex. Annual Review of Neuroscience, 28(1), 377-401.

Patel, A. (2008). Music, language, and the brain. Oxford: Oxford University Press.

Patel, A. (2011). Why would musical training benefit the neural encoding of speech? The opera Hypothesis. Frontiers in Psychology, 2. doi: 10.3389/fpsyg.2011.00142.

Piaget, J. (2008). Intellectual Evolution from Adolescence to Adulthood. Human Development, 51(1), 40-47. doi: $10.1159 / 000112531$.

Sacks, O. (2007). Musicophilia. Nova York: Alfred A. Knopf. Salimpoor, V., Benovoy, M., Larcher, K., Dagher, A. e Zatorre, R. (2011). Anatomically distinct dopamine release during anticipation and experience of peak emotion to music. Nature Neuroscience, 14(2), $257-$ 262. doi: $10.1038 / \mathrm{nn} .2726$

Samanez-Larkin, G. R. e Carstensen, L. L. (2011). Socioemotional functioning and the aging brain. Em J. Decety e J. T. Cacioppo (Eds.), The Oxford Handbook of Social Neuroscience (pp. 507-521). Nova York: Oxford University Press.

Shifriss, R., Bodner, E. e Palgi, Y. (2014). When you're down and troubled: Views on the regulatory power of music. Psychology of Music, 43(6), 793-807. doi: 10.1177/0305735614540360.

Sprinthall, N. A. (1991). Role-talking programs for high-school student: new methods to promote psychological development. Em B. P. Campos (Ed.), Psychological intervention and human development (pp. 33-38). Porto, Portugal: Instituto de Consulta Psicológica Formação e Desenvolvimento.

Van Goethem, A. e Sloboda, J. (2011). The functions of music for affect regulation. Musicae Scientiae, 15(2), 208-228. doi: 10.1177/1029864911401174.

Valquaresma, A. e Coimbra, J. L. (2013). Criatividade e educação: A educação artística como o caminho do futuro? Educação, Sociedade \& Culturas, 40, 131-146. Recuperado de https://goo.gl/AJkHHY.

Vaz, F. e Martins, C. (2009). Diferenciação e Regulação Emocional na Idade Adulta: Tradução e validação de dois 
instrumentos de avaliação para a população portuguesa (dissertação de mestrado). Instituto de Educação e Psicologia, Universidade do Minho, Braga, Portugal.

Vygotsky, L. (1971). The psychology of art. Cambridge, Mass.: M.I.T. Press.
Zahn-Waxler, C., Radke-Yarrow, M. e Brady-Smith, J. (1977). Perspective-taking and prosocial behavior. Developmental Psychology, 13(1), 87-88. doi: 10.1037/00121649.13.1.87.

Zull, J. (2002). The art of changing the brain. Sterling, Va.: Stylus Pub. 